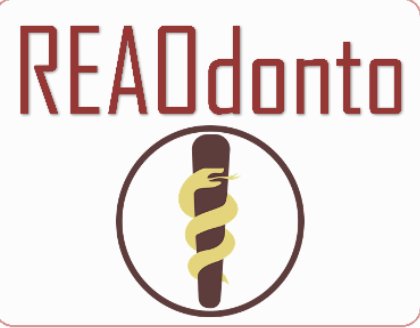

Revista Eletrônica Acervo Odontológico

\section{REVISÃO BIBLIOGRÁFICA}

Recebido em: 10/2020

Aceito em: 11/2020

Publicado em: 12/2020

\title{
Selamento de dentina cariada: uma alternativa minimamente invasiva
}

\author{
Cariated dentine sealing: a minimally invasive alternative
}

Sellado dentino cariado: una alternativa mínimamente invasiva

\begin{abstract}
Zildenilson da Silva Sousa ${ }^{1}$, Maria Isabelly Pessoa Brandão ${ }^{1}$, Iolanda Maria Marques de Lima ${ }^{1}$, Cleginaldo Torres de Mesquita ${ }^{1}$, Lorrana da Silva Pereira ${ }^{1}$, Eduardo da Cunha Queiroz ${ }^{2}$, Karlos Eduardo Rodrigues Lima ${ }^{2}$, Iana Sá de Oliveira Fontenelle ${ }^{1,3}$, Juliana Araújo Oliveira ${ }^{1,3}$, Talita Arrais Daniel Mendes ${ }^{1,3 *}$.
\end{abstract}

\begin{abstract}
Resumo: Este estudo objetivou realizar uma revisão de literatura acerca do uso de selamento de cárie em dentina como uma alternativa minimamente invasiva. Para tanto, foi realizada uma busca bibliográfica nas bases de dados PubMed e Science Direct, utilizando os descritores "Fissure Sealants" e "Dental cárie" combinados entre si pelo operador booleano AND. Chegou-se a um total de 214 artigos encontrados, que após uma leitura crítica de títulos e resumos foram selecionados 12 destes, sendo todos na língua inglesa e publicados nos últimos 10 anos. Os resultados obtidos por meio da literatura evidenciaram a eficácia dos materiais seladores em lesões superficiais de cárie em dentina, levando em consideração fatores como a localização da lesão com abertura máxima de 3 milímetros e com cavidade de média profundidade. A aplicação desta técnica tem sido eficaz, no entanto, faz-se necessário a realização de mais estudos a fim de melhor elucidar a sua prática e atividade clínica.
\end{abstract}

Palavras-chave: Selantes de fossas e fissuras, Cárie dentária, Dentina.

\begin{abstract}
This study aimed to conduct a literature review about the use of dental caries sealing as a minimally invasive alternative. For this purpose, a bibliographic search was performed in the PubMed and Science Direct databases, using the descriptors "Fissure Sealants" and "Dental caries" combined by the Boolean operator AND. A total of 214 articles were found, which after a critical reading of titles and abstracts, 12 of these were selected, all in English and published in the last 10 years. The results obtained through the literature showed the efficacy of the sealing materials in superficial lesions of caries in dentin, taking into account factors such as the location of the lesion with a maximum opening of $3 \mathrm{~mm}$ and with a medium depth cavity. The application of this technique has been effective, however, it is necessary to carry out further studies in order to better elucidate its practice and clinical activity.
\end{abstract}

Keywords: Pit and fissure sealants, Dental caries, Dentin.

Resumen: Este estudio tuvo como objetivo realizar una revisión de la literatura sobre el uso del sellado de caries dental como alternativa mínimamente invasiva. Para ello, se realizó una búsqueda bibliográfica en las bases de datos PubMed y Science Direct, utilizando los descriptores "Fissure Sealants" y "Dental caries"

${ }^{1}$ Centro Universitário Maurício de Nassau (UNINASSAU), Fortaleza - CE.

2 Centro Universitário Católica de Quixadá (Unicatólica), Quixadá - CE.

3 Universidade Federal do Ceará (UFC), Fortaleza - CE. *E-mail: talita_arrais@hotmail.com 
combinados por el operador booleano AND. Se encontraron un total de 214 artículos, que luego de una lectura crítica de títulos y resúmenes, se seleccionaron 12 de estos, todos en inglés y publicados en los últimos 10 años. Los resultados obtenidos a través de la literatura demostraron la eficacia de los materiales de sellado en lesiones superficiales de caries en dentina, teniendo en cuenta factores como la ubicación de la lesión con una apertura máxima de $3 \mathrm{~mm}$ y con una cavidad de profundidad media. La aplicación de esta técnica ha sido eficaz, sin embargo, es necesario realizar más estudios con el fin de dilucidar mejor su práctica y actividad clínica.

Palabras clave: Selladores de fosas y fisuras, Caries dental, Dentina.

\section{INTRODUÇÃO}

A cárie dentária é uma doença definida como uma pandemia global, que com o consumo de açúcar e fatores inadequados de higiene bucal podem contribuir para o aparecimento dessas lesões em elementos dentários (MONSE B, et al., 2012). Em estágios iniciais, é tida como uma etologia identificada, dependente de fatores secundários, dos quais influenciam na estagnação do biofilme na superfície do dente, gerando uma placa de bactéria capaz de progredir com doença, gerando, por conseguinte a desmineralização como o resultado dinâmico deste processo infeccioso (HESSE D, et al., 2014).

A maneira terapêutica de como o tratamento de lesões de cárie ativa em elemento dentário deve ser intervinda pode variar, sendo o diagnóstico precoce fundamental para intervir de maneiras micro invasivas (BORGES BC, et al., 2010).

Convencionalmente, a lesão de cárie é eliminada através de procedimentos operacionais que visam reestabelecer a integridade daquela determinada superfície (HESSE D, et al., 2014). Estes tratamentos tradicionais consistem na remoção de lesões cariosas por completo antes do procedimento restaurador, porém, o preparo dessas cavidades gera uma grande perda de tecidos saudáveis (ALVES LS, et al., 2017). Esses procedimentos são considerados invasivos, com uso de perfuração e desgaste do elemento dentário (DORRI M, et al., 2015).

Se tratando de cárie de esmalte, a possibilidade de paralisação de progressão de doença por meio de vias não invasivas é recomendada pela literatura, todavia, em dentina a aplicação de procedimentos invasivos ainda é utilizada pelos cirurgiões-dentistas (BORGES BC, et al., 2010).

As abordagens de técnicas alternativas têm sido proposta com o intuito de preservar a estrutura do dente, através do selamento de estruturas cariadas com selantes ou materiais resinosos dos quais tem a capacidade de controlar e regredir a doença de maneira significativa, eliminando os micro-organismos infecciosos presente na estrutura (ALVES LS, et al., 2017).

Os selantes são desenvolvidos a base de resinas e tem como objetivo proteger determinadas superfícies suscetíveis de desenvolver lesões de cárie, recobrindo fossas e fissuras, agindo como uma camada protetora que evita a retenção de determinados agentes que contribuem para o aparecimento dessas lesões (HESSE D, et al., 2014). São compostos de bisfenol metacrilato de glicidila (bis-GMA), que são endurecidos ao ser exposto a luz, sua aplicação é na superfície dentária obliterando fisicamente o sistema de fossas e fissuras e com isso elimina-se possíveis foco de contaminação da bactéria Streptococcus mutans (CHESTNUTT IG, et al., 2017).

Evidências defendem que a aplicação de selantes traz benefícios sendo esses utilizados como uma abordagem preventiva na odontologia, contudo, em um elemento com dentina já cariado, a remoção completa da dentina não é necessária, assim essa medida de aplicação demostra resultados positivos na prática clínica (HESSE D, et al., 2014). Outros materiais como resinas fluidas e cimentos de ionômero de vidro tem aplicabilidade com a mesma função de selamento em dentina cariada (DORRI M, et al., 2015). O selamento de elemento dentário visa a paralização do processo carioso por meio da formação de uma barreira mecânica hermeticamente selada que impede a retenção de alimentos e dessa forma a progressão da cárie, além de melhorar a higienização local preservando o renascente dentário, além de ser uma alternativa rápida e de baixo custo (ALVES LS, et al., 2017). 
No entanto, ainda não há consenso da eficácia do selamento de tecido cariado, quanto ao sucesso e eficácia dos materiais utilizados, assim sendo, o objetivo deste estudo é revisar a literatura acerca dos materiais de prevenção de cárie em dentina, identificando medidas minimamente invasivas de selamento de cárie.

\section{MÉTODOS}

Trata-se de uma revisão da literatura, onde realizou-se a busca na plataforma de dados da PUBMED e Science Direct, aplicou-se os descritores cadastrados no Descritores em Ciências da Saúde (DeCS): "Fissure Sealants" e "Dental cárie", na língua inglesa e interligados pelo operador booleano "AND". Realizou-se um levantamento bibliográfico dos últimos 10 anos (período entre 2010 a 2020), o total de artigos encontradas foi de 214. Após uma leitura, foram selecionados 12.

Os critérios de inclusão adotados foram artigos originais que tinham como temática ensaios clínicos, testes controlados e aleatórios com resultados significativos. Excluiu-se modelos de revisão de literatura, monografias, relatos de caso e estudos que não estavam diretamente relacionados a temática, além daqueles que evidenciavam procedimentos invasivos na dentina em decorrência de elementos dentários com cárie extensa (Figura 1).

Figura 1 - Fluxograma de busca e seleção dos artigos.

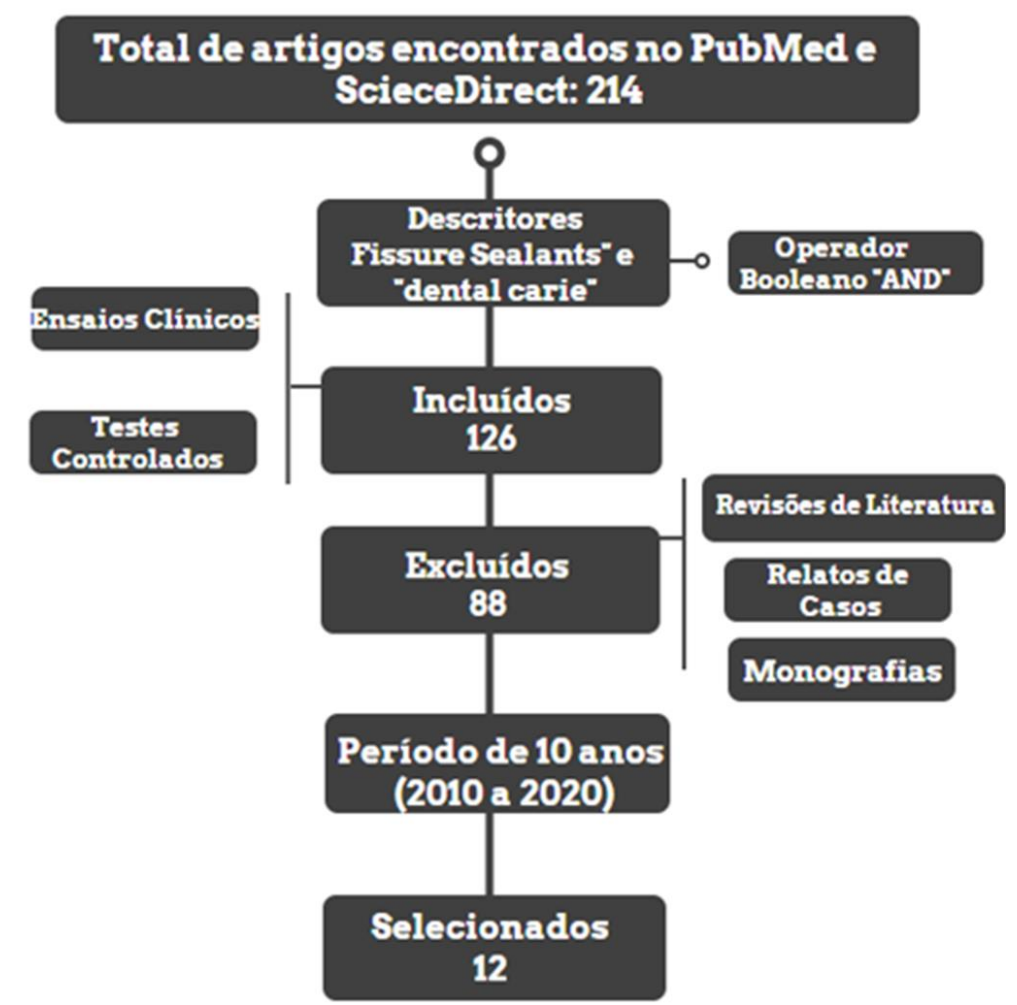

Fonte: SOUSA ZS, et al., 2020.

\section{RESULTADOS}

Após a busca por artigos que explicassem os assuntos em diferentes aspectos, procurou-se nesta revisão de literatura juntá-los em uma tabela, a fim de melhorar o entendimento dos resultados alcançados. Obtiveram-se 9 ensaios clínicos randomizados, 1 revisão sistemática, 1 estudo laboratorial in vitro e 1 metaanálise.

Os estudos confirmaram a relevância da terapia de selamento de cárie em dentina nesta perspectiva de tratamento de cárie dentinária (Tabela 1). 
Tabela 1 - Resultados da pesquisa bibliográfica.

\begin{tabular}{|c|c|c|c|c|}
\hline Autor / ano & Tipo de estudo & Grupos & Métodos & Resultados \\
\hline FEI X, et al., 2020 & In vitro & $\begin{array}{c}\text { Selante contendo } \mathrm{CaF}_{2} \\
\text { nanopartículas(nCaF2) e metacrilato } \\
\text { de dimetilamino-hexadecil } \\
\text { (DMAHDM); Helioseal F utilizado } \\
\text { como controle. }\end{array}$ & $\begin{array}{l}\text { Resistência ao cisalhamento; } \\
\text { dureza e liberação de íons F } \\
\text { foram medidas; teste } \\
\text { microbiológico para } \\
\text { Streptococcus mutans }\end{array}$ & $\begin{array}{l}\text { Promissor na inibição de cárie e } \\
\text { promoção da remineralização do } \\
\text { esmalte e da dentina. }\end{array}$ \\
\hline $\begin{array}{l}\text { ALSABEK L, et } \\
\text { al., } 2019\end{array}$ & Ensaio Clínico & $\begin{array}{c}\text { Grupo A: } \\
\text { Embrace }^{\mathrm{TM}} \text { WetBond }{ }^{\mathrm{TM}} \\
\text { Grupo B } \\
\text {-Fuji TRIAGE® }\end{array}$ & $\begin{array}{l}\text { Retenção dos materiais foi } \\
\text { analisada aos } 3 \text { e } 6 \text { meses }\end{array}$ & $\begin{array}{l}\text { Lesões de cárie oclusal, restritas ao } \\
\text { esmalte, podem ser contidas por } \\
\text { selamento da lesão com selante de } \\
\text { resina hidrofílica e selante de } \\
\text { ionômero de vidro. }\end{array}$ \\
\hline $\begin{array}{l}\text { ZHANG W, et al, } \\
2019\end{array}$ & Ensaio clínico & $\begin{array}{l}\text { - Carbômero de vidro } \\
\text { - Composto de resina }\end{array}$ & $\begin{array}{l}\text { Lesões de cárie no esmalte e } \\
\text { dentinária microcavitadas em } \\
\text { fossas e fissuras dos } \\
\text { primeiros molares } \\
\text { permanentes }\end{array}$ & $\begin{array}{c}\text { Lesões de dentina com um pequeno } \\
\text { orifício }(\varnothing<0,5 \mathrm{~mm}) \text { em fossas e } \\
\text { fissuras de superfícies oclusais em } \\
\text { molares permanentes podem } \\
\text { sobreviver por quatro anos se forem } \\
\text { seladas. }\end{array}$ \\
\hline $\begin{array}{l}\text { DIAS KR, et al, } \\
2018\end{array}$ & Ensaio clínico & $\begin{array}{l}\text { Grupo teste: Cárie com resina fluida } \\
\qquad(\mathrm{SC}) \text {. } \\
\text { Grupo controle: Remoção parcial da } \\
\text { cárie seguida por restauração (RPC). }\end{array}$ & $\begin{array}{l}\text { Lesões de cárie em molares } \\
\text { decíduos, restaurações } \\
\text { avaliadas pelos critérios do } \\
\text { USPHS e análise } \\
\text { radiográfica. }\end{array}$ & $\begin{array}{c}\text { Selamento de dentina cariada reduz o } \\
\text { tempo de cadeira e demonstra taxa de } \\
\text { sucesso clínico. Nenhuma diferença } \\
\text { radiográfica em relação à remoção } \\
\text { parcial da cárie seguida de } \\
\text { restauração. }\end{array}$ \\
\hline $\begin{array}{l}\text { DE AMORIM RG, } \\
\text { et al., } 2018\end{array}$ & Revisão sistemática & $\begin{array}{l}\text {-PubMed } \\
\text {-PubMed Central } \\
\text {-Google Scholar }\end{array}$ & $\begin{array}{c}\text { Seis revisores independentes } \\
\text { selecionaram } 43 \text { publicações } \\
\text { de um total de } 1958 \text { estudos } \\
\text { potencialmente relevantes } \\
\text { recuperados. }\end{array}$ & $\begin{array}{l}\text { Os selantes ART apresentaram um } \\
\text { alto efeito de prevenção de cárie. }\end{array}$ \\
\hline
\end{tabular}

REAOdonto | Vol. 2 | e5743 | DOI: https://doi.org/10.25248/REAOdonto.e5743.2020 Página 4 de 10 
Revista Eletrônica Acervo Odontológico / Electronic Journal Dental Collection | ISSN 2674-7200

\begin{tabular}{|c|c|c|c|c|}
\hline Autor / ano & Tipo de estudo & Grupos & Métodos & Resultados \\
\hline $\begin{array}{c}\text { CHESTNUTT IG, } \\
\text { et al., } 2017\end{array}$ & Ensaio clínico & $\begin{array}{c}\text { Grupo 1: Recebeu selantes para } \\
\text { fissura (FS) } \\
\text { Grupo 2: Recebeu verniz fluoretado } \\
\text { (FV) }\end{array}$ & $\begin{array}{l}\text { Nível de cárie na linha de } \\
\text { base de sexo e dentição } \\
\text { primária, usando a } \\
\text { minimização na proporção de } \\
\text { 1: } 1 \text { para os tratamentos. }\end{array}$ & $\begin{array}{l}\text { Resultados semelhantes quando } \\
\text { examinados os números de dentes } \\
\text { cariados. }\end{array}$ \\
\hline $\begin{array}{c}\text { ALVES LS, et al., } \\
2017\end{array}$ & Ensaio clínico & $\begin{array}{c}\text { Teste: selante inseridos diretamente } \\
\text { sobre a lesão cariosa } \\
\text { Controle: tratamento restaurador } \\
\text { convencional. }\end{array}$ & $\begin{array}{l}\text { Presença de cárie oclusal em } \\
\text { molares permanentes e pré- } \\
\text { molares, avaliada por } \\
\text { radiografia bitewing. }\end{array}$ & $\begin{array}{l}\text { O selamento de lesões de cárie } \\
\text { oclusal em dentes permanentes } \\
\text { conseguiu controlar a cárie por um } \\
\text { período de 3-4 anos. }\end{array}$ \\
\hline $\begin{array}{l}\text { DORRI M, et al, } \\
2015\end{array}$ & Meta-análise & $\begin{array}{l}\text {-Tratamento micro invasivo } \\
\text {-Tratamento não invasivo (exemplo, } \\
\text { verniz de flúor). }\end{array}$ & $\begin{array}{l}\text { Utilizando o método Becker- } \\
\text { Balagtas para calcular a } \\
\text { razão de chances (OR) para } \\
\text { a progressão da lesão e } \\
\text { métodos GRADE. }\end{array}$ & $\begin{array}{l}\text { O tratamento micro invasivo de lesões } \\
\text { de cárie proximal é eficaz e tem um } \\
\text { benefício significativo sobre } \\
\text { intervenções não-invasivas. }\end{array}$ \\
\hline $\begin{array}{l}\text { HESSE D, et al., } \\
2014\end{array}$ & Ensaio clínico & $\begin{array}{l}\text { Teste: Selante à base de resina de } \\
\text { fossas e fissuras aplicados sem } \\
\text { remoção de tecido cariado. Controle: } \\
\text { Tratamento restaurador com } \\
\text { compósito de resina com remoção } \\
\text { parcial. }\end{array}$ & $\begin{array}{l}\text { Avaliação de cárie de acordo } \\
\text { com pontuações ICDAS e } \\
\text { atividades visuais de acordo } \\
\text { com os critérios tátil de } \\
\text { Nyvad }\end{array}$ & $\begin{array}{l}\text { Nenhuma progressão de cárie foi } \\
\text { registrada nas avaliações } \\
\text { radiográficas. }\end{array}$ \\
\hline
\end{tabular}

REAOdonto | Vol. 2 | e5743 | DOI: https://doi.org/10.25248/REAOdonto.e5743.2020 Página 5 de 10 


\begin{tabular}{|c|c|c|c|c|}
\hline Autor / ano & Tipo de estudo & Grupos & Métodos & Resultados \\
\hline $\begin{array}{c}\text { MARKOWITZ K, } \\
\text { et al., } 2013\end{array}$ & Ensaio clínico & $\begin{array}{c}\text {-Selantes transparentes (Shofu Clear } \\
\text { ou Delton não preenchidos) } \\
\text {-Selantes opacos (3M Clinpro ou } \\
\text { Delton FS) }\end{array}$ & $\begin{array}{l}\text { Calculada a sensibilidade e } \\
\text { especificidade das medidas } \\
\text { da câmera florescente na } \\
\text { detecção de cárie dentinária } \\
\text { de dentes extraídos, antes e } \\
\text { após a aplicação de selantes }\end{array}$ & $\begin{array}{l}\text { Houve uma perda significativa de } \\
\text { sensibilidade na detecção de cárie } \\
\text { dentinária. }\end{array}$ \\
\hline $\begin{array}{c}\text { MONSE B, et al., } \\
2012\end{array}$ & Ensaio clínico & $\begin{array}{l}\text {-Diamino fluoreto de prata } \\
\text {-Selante de ionômero de vidro } \\
\text { - Sem tratamento clínico }\end{array}$ & $\begin{array}{c}\text { Avaliada a taxa de retenção } \\
\text { dos selantes em um período } \\
\text { de } 18 \text { meses }\end{array}$ & $\begin{array}{c}\text { Selantes de ATR com ionômero de } \\
\text { vidro reduziram significativamente o } \\
\text { aparecimento de novas lesões de cárie } \\
\text { dentinária }\end{array}$ \\
\hline $\begin{array}{l}\text { BORGES BC, et } \\
\text { al., } 2010\end{array}$ & Ensaio clínico & $\begin{array}{l}\text { Experimental: recebeu aplicação do } \\
\text { selante Fluorshield (Dentsply) } \\
\text { Controle: sem intervenção clínica }\end{array}$ & $\begin{array}{l}\text { Integridade marginal do } \\
\text { selante foi avaliada no grupo } \\
\text { experimental. }\end{array}$ & $\begin{array}{l}\text { Progressão da cárie clínica e } \\
\text { radiográfica foi significativamente mais } \\
\text { frequente nos dentes controle quando } \\
\text { comparados ao grupo experimental ( } P \\
<0,05)\end{array}$ \\
\hline
\end{tabular}

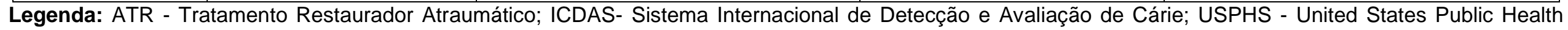
Service.

Fonte: SOUSA ZS, et al., 2020. 


\section{DISCUSSÃO}

Os dados coletados por meio da literatura demostram resultados positivos na aplicação de selantes em elemento dentário com tecido de dentina cariado como medida minimante invasiva de contenção, visando preservar o máximo de tecido saudável, além de eliminar ou reduzir por um período os micro-organismos que afetam o tecido dentinário. Alguns estudos realizaram uma comparação com dois tipos de lesões de cárie presente no elemento dentário. O ensaio clínico de Hesse D, et al. (2014), com 36 crianças de idade entre 4 a 9 anos, com lesões de cárie ativas e inativas, afirma a eficácia dos selantes a base de resina de fossa e fissura, que em um período de 18 meses não demostraram progressão das lesões em tecido dentinário, isso pode-se dar ao fato de a união de resina ser bastante favorável em esmalte, onde se necessita de uma selamento de margens eficaz.

Hesse D, et al. (2014), afirmam ainda que esse procedimento de selamento em elemento dentário evidencia vantagens, pois além de ser um tratamento minimamente invasivo, reduz significativamente 0 tempo de tratamento quando comparado a procedimentos restauradores de técnica convencional.

Nesse mesmo sentido, o estudo de Monse B, et al. (2012), que teve o mesmo período de duração que de Hesse D, et al. (2014), obteve-se uma amostra significativa de 704 crianças em idades escolar variáveis de 6 a 12 anos. Todavia, os materiais utilizados no estudo de Monse B, et al. (2012) foram os selantes para tratamento restaurador atraumático (ART), no qual pode-se comprovar sua eficácia na progressão dessas lesões, o que torna uma alternativa viável quando se tem populações onde não tem acesso à uma estrutura física de consultório odontológico.

Para Fei X, et al. (2020), essa lesão de cárie dentária pode ser definida como uma doença oral crônica que se desenvolve a partir de acúmulo de biofilme e exposição a açúcar na superfície dentária e que afeta atualmente a maioria das populações em todo o mundo. Nesse viés, Markowitz K, et al. (2014), relata que o monitoramento de dentes selados para evidências de início e progressão desta doença oral pode ser desafiadora, particularmente em dentes tratados com selantes opacos, no qual impedem a visualização direta da anatomia oclusal dentária.

Nesse sentido, diversas tecnologias complementares podem ser utilizadas para evidenciar a eficácia do tratamento através desses materiais aplicados em dentina cariada. Desse modo, Markowitz K, et al. (2014) através de seu estudo in vitro utilizou o Spectra Caries Detection Aid, um dispositivo detector de cárie com câmera fluorescente (FC) que tem a capacidade de detectar cáries oclusais que se estendem para a dentina com boa sensibilidade e especificidade para um diagnóstico mais acurado. E usaram agentes de selamento translúcidos da Shofu Clear ou Delton, bem como selantes opacos 3M Clinpro ou Delton FS, concluindo que a aplicação de selante sem cor causou uma perda insignificante de sensibilidade de detecção de integridade de processo restaurador, o que pode dificultar a avaliação e o procedimento clínico, assim sendo para melhor análise de um selamento mais efetivo preconiza-se materiais com opacidade (MARKOWITZ K, et al., 2013).

Nesse sentido, Alsabek L, et al. (2019), visando identificar de uma maneira mais precisa o efeito de retenção e remineralização do selante a base de resina tolerante à umidade e selante de ionômero de vidro em cáries não cavitadas e fissuras, utilizou o dispositivo DIAGNOdent (Kavo®, Biberach, Alemanha) em seu ensaio clínico como forma de obter-se leitura e realizar as gravações das mesmas. Os mesmos afirmam que lesões de cárie oclusal, restrita ao nível de tecido de esmalte podem ser tratadas com apenas a aplicação de selantes de resina hidrofílica e selante de ionômero de vidro.

Alsabek L, et al. (2019) complementam afirmando que o Embrace TM WetBond ${ }^{\mathrm{TM}}$ mostrou-se superior a nível de retenção quando comparado ao grupo que recebeu selante de ionômero de vidro em um período de 6 meses de avaliação, podendo -se atentar ao fato de que o ionômero de vidro pode não ser um material de primeira escolha nessa perspectiva.

Chestnutt IG, et al. (2017) concluiu através de um ensaio clínico com amostra total de 1.016 crianças randomizadas de faixa etária de 6 e 7 anos de idade que através da aplicação de selante de fissuras (FS) e verniz fluoretado (FV) demonstrou ser eficaz e que após os 36 meses de estudos, não houve diferença 
significativa em relação a aplicação de FV quando comparado com a manutenção com FS. Assim sendo, o FV demonstrou ser melhor em relação a benefício económicos se tratando na prevenção ou contenção de cárie (DIAS KR, et al. 2018; CHESTNUTT IG, et al. 2017)

Contudo, a eficácia do tratamento com selantes pode ser variável conforme a localização em que a lesão se encontra (BORGES BC, et al. 2010). Assim, Alves LS, et al. (2017), através de avaliação clínica e radiográfica de lesões de cárie em terço externo de dentina em 49 pacientes de 8 a 43 anos, avaliados em um período de 3 a 4 anos, demostrou que não há resultados significativos entre os grupos quando comparado a aplicação de selantes nessa região a métodos restauradores com resina. Por outro lado, Alves LS, et al. (2017) afirmam que o uso de selantes como uma técnica de controle de cáries oclusais cavitadas pode evitar ou adiar possíveis procedimentos com materiais restauradores, apesar de a dentina se tornar mais mineralizadora ao longo do tempo e reduzir na remoção de tecido.

Para Fei X, et al. (2020), a morfologia complexa de fossas e fissuras dentais tornam muito mais suscetíveis a cárie, quando comparada a superfícies lisas, sendo que os biofilmes nestes locais podem produzir ácidos orgânicos que podem causar a perda de minerais. Assim sendo, o selamento da dentina cariada auxilia no controle da sensibilidade dos dentes, agindo como uma barreira protetora entre 0 ambiente oral e a dentina sob esmalte não cavitada fornecida pelo próprio agente selador (BORGES BC, et al. 2010). Se tratando de selar cárie oclusal em dentes permanentes é possível controla a lesão durante este período de tempo, contribuindo para melhora no prognóstico do dente (ALVES LS, et al. 2017).

Nessa perspectiva, Borges BC, et al. (2010), em um ensaio clínico realizado com a divisão de dois grupos de 30 dentes cada, sendo um submetido a tratamento com selante de fossa e fissura Fluorshield (Dentsply) e outro sem qualquer tratamento clínico, concluiu que lesões de cárie oclusais não cavitadas em tecido dentinário são de difícil diagnostico, e para que haja uma melhor evidenciação dessas lesões é importante que se utilize de equipamentos como o detector de cárie de diodo a laser em conjunto com exames de imagem tradicionais.

Assim, esse tipo de selante a base de resina demonstra boa resistência a possíveis fatores que possam contribuir para o aparecimento de lesões, retendo a estrutura dentária e aumentando a resistência ao desgaste, agindo como uma barreira protetora contra o biofilme (BORGES BC, et al., 2010). Analisando os estudos que comparam grupos teste com aplicação de selante a grupos controle sem tratamentos clínicos, os selantes apresentam uma alta taxa de contenção da progressão de cárie de dentina em superfície oclusais de primeiros molares nos estudos avaliados (BORGES BC, et al., 2010). Complementando, De Amorin RG, et al. (2018) através de sua revisão sistemática com meta-análise de 43 publicações evidenciaram em seu estudo a eficácia de alto efeito preventivo na aplicação de selantes de ART em elementos dentários, assim, os autores destacaram que esses selantes e restaurações são ideias para o tratamento e prevenção de lesões de cárie tanto em dentes posteriores quanto inferiores primários e permanentes.

De Amorin RG, et al. (2018), evidenciam ainda que em comparação com os materiais restauradores comumente utilizados pelos cirurgiões-dentistas, como o caso das restaurações de tratamento restaurador atraumático (ART) apresenta alta taxa de sobrevida, diferentemente dos selantes de ART. Desse modo, Para Dorri M, et al. (2015), a utilização de tratamentos micro invasivos reduzem as chances de progressão de lesão de cárie quando comparado com o tratamento não invasivo como o verniz fluoretado e também instruções de higiene oral adequada, com a utilização de fio dental.

Nesse sentido, o ensaio clínico de Zhang W, et al. (2019) obteve uma amostra significativa de 405 crianças de idade média de 8 anos, alocadas em grupos compostos de vidro de alta viscosidade, método de tratamento restaurativo atraumático (HVGIC/ART), carbômero de vidro e composto de resina no qual observou a progressão de lesões cariosas em covas micro-cavitadas em dentina seladas e fissuras em comparação a poços selados e fissuras sem lesões cariosas ou esmaltes na linha de base. Zhang W, et al. (2019) concluiu que progressão das micro cavidades seladas com HVGIC de acordo com o método ART, durante um período de quatro anos é baixa e as lesões de dentina com um pequeno orifício $(\varnothing<0,5 \mathrm{~mm})$ em fossas e fissuras podem sobreviver por quatro anos se forem seladas. 
Em contrapartida, Dias KR, et al. (2018), em seu estudo através de resina fluida em dente cariado, com a presença de lesão de extensão até a metade externa do tecido dentinário, realizado em um período de 2 anos de acompanhamento com 28 crianças, de idades entre 3 a 8 anos com molares decíduos, totalizando 57 elementos dentários, demonstrou não haver diferenças entre grupo teste e controle, assim, os autores concluem que selamento de dentina cariada pode ser aplicada na odontologia visando uma alternativa minimante invasiva de contenção, em decorrência de seu sucesso clínico.

Contudo, Dorri M, et al. (2015), diferentemente de Dias KR, et al. (2018), ressalva através de sua revisão sistemática com meta-análise de 8 ensaios clínicos que randomizaram 365 participantes que não está claro ainda quais desses tratamentos micro invasivos demonstra-se mais vantajosos ou adequado, sendo que isso pode variar em decorrência de fatores complementares. Apesar disso, os autores concluem que através das evidências disponíveis, o tratamento micro invasivo de lesões de cárie proximal detém o esmalte não cavitado e as lesões dentinárias iniciais, limitadas ao terço externo da dentina, com base em exames de imagem, com a radiografia.

Por conta disso, a odontologia está sempre atualizando-se, visando estratégias de tratamento minimante invasivas. Assim, Fei X, et al. (2020), através de estudo in vitro avaliou o desenvolvimento e eficácia de um novo selante composto por CaF2nanopartículas ( $\mathrm{nCaF} 2$ ) e metacrilato de dimetilamino-hexadecil. Fei $\mathrm{X}$, et al.(2020) em seu estudo observou também e os efeitos desses selantes sobre os biofilmes dentários criados pela bactéria Streptococcusmutans, avaliando a dureza do material e a liberação de íons, além de testes microbiológicos, obtendo como resultados, fortes propriedades antibacterianas que com a liberação significativa de flúor houve resultados positivos relevantes $(p<0,05)$ a inibição de cárie e remineralização de esmalte e dentina, tornando-se segundo o autor uma boa opção de tratamento dessa doença.

\section{CONSIDERAÇÕES FINAIS}

Verificou-se que os estudos indicam efeitos positivos do selamento dentário com dentina afetada por cárie. Além de ser um tratamento minimamente invasivo, reduz significativamente o tempo de tratamento quando comparado a procedimentos restauradores de técnica convencional, promovendo a eficácia da redução e paralização doença, contribuindo ainda para a redução na perda de tecido saudável que seria ocasionado nestes processos restauradores invasivos. Apesar disso, é importante a realização de mais estudos em um período e números amostrais maiores para que possam ser estabelecidos a ligação e eficácia entre diversos tipos de selantes associados a hábitos de higiene e a fatores fisiológicos que afetem a saúde da cavidade bucal. Vale salientar que este estudo contribui de maneira positiva para demonstrar a comunidade de Cirurgiões-dentistas a importância da preservação dos tecidos dentários com novas técnicas relativamente simples de se executar.

\section{AGRADECIMENTOS}

Agradecimento ao Centro Universitário Maurício de Nassau - Fortaleza, Ceará.

\section{REFERÊNCIAS}

1. ALSABEK L, et al. Retention and remineralization effect of moisture tolerant resin-based sealant and glass ionomer sealant on non-cavitated pit and fissure caries: Randomized controlled clinical trial. J Dent, 2019; 86: 69-74.

2. ALVES LS, et al. A randomized clinical trial on the sealing of occlusal carious lesions: 3-4-year results. Braz Oral Res, 2017; $31: e 44$.

3. BORGES BC, et al. Efficacy of a pit and fissure sealant in arresting dentin non-cavitated caries: a 1-year follow-up, randomized, single-blind, controlled clinical trial. Am J Dent, 2010; 23(6): 311-316.

4. CHESTNUTT IG, et al. Seal or Varnish? A randomised controlled trial to determine the relative cost and effectiveness of pit and fissure sealant and fluoride varnish in preventing dental decay. Health Technol Assess, $2017 ; 21(21): 1-256$.

5. DE AMORIM RG, et al. Survival percentages of atraumatic restorative treatment (ART) restorations and sealants in posterior teeth: an updated systematic review and meta-analysis. Clin Oral Investig, 2018; 22(8): 2703-2725. 
6. DIAS KR, et al. Efficacy of sealing occlusal caries with a flowable composite in primary molars: A 2-year randomized controlled clinical trial. J Dent, 2018; 74: 49-55.

7. DORRI M, et al. Micro-invasive interventions for managing proximal dental decay in primary and permanent teeth. Cochrane Database Syst Ver, 2015; (11): CD010431.

8. FEI X, et al. Novel pit and fissure sealant containing nano-CaF2 and dimethylaminohexadecyl methacrylate with double benefits of fluoride release and antibacterial function. Dental Materials : Official Publication of the Academy of Dental Materials, 2020; 36(9): 1241-1253.

9. HESSE D, et al. Sealing versus partial caries removal in primary molars: a randomized clinical trial. BMC Oral Health, $2014 ; 14: 58$.

10. MARKOWITZ K, et al. Effect of pit and fissure sealants on caries detection by a fluorescent camera system $\mathrm{J}$ Dent, 2013; 41 (7): 590-599.

11. MONSE B, et al. Caries preventive efficacy of silver diammine fluoride (SDF) and ART sealants in a school-based daily fluoride toothbrushing program in the Philippines. BMC Oral Health, 2012; 12: 52.

12. ZHANG W, et al. Is preventing micro-cavities in dentine from progressing with a sealant successful?. Braz Dent $\mathrm{J}$, 2019; 226 (8): 590-594. 